\title{
La science et le scientifique: confronter les points de vue sur les explorations d'Alfred Grandidier
}

Jehanne-Emmanuelle Monnier

\section{Summary}

This article embraces the different ways a scientist traveller and his fieldworks can be perceived through the case of Alfred Grandidier in South America, in India and in Madagascar (1857-1870). Our aim is to deal with various aspects of historical scientific exploration and to draw a picture of the erudite traveller by crossing various and complementary points of view. Scientific works can be received very differently, depending on the place and the nature of the addresses, whether they are authorities wishing to take advantage of it, native people fearing for their safety or disciples glorifying their idol. The concept of science itself and the status of the scientist raise the question of mutual understanding between the explorer and his contemporaries, in Paris or in a remote place. Alfred Grandidier's example is particularly relevant to reveal different visions of scientist traveller as promoted in the 19th century, as well as the various perceptions that a scientific work can have depending on the public it impacts.

Keywords: Science, exploration, fieldworks, perception, Alfred Grandidier

\section{Résumé}

Cet article confronte les regards portés sur un voyageur-scientifique et ses travaux de terrain à travers le cas d'Alfred Grandidier en Amérique du Sud, en Inde et à Madagascar (1857-1870). Il s'agit d'approcher les multiples aspects de la science en situation d'exploration et de cerner le portrait du savant voyageur en croisant des points de vue très divers et complémentaires. La réception des travaux scientifiques varie considérablement selon les lieux

Jehanne-Emmanuelle Monnier, Université de La Réunion, CRESOI (Centre de recherches et d'études sur les sociétés de l'Océan Indien), (jem@immram.com). 
et les individus, qu'il s'agisse des autorités souhaitant en tirer un parti immédiat, des peuples autochtones craignant pour leur sécurité ou des héritiers intellectuels élevant un panthéon en l'honneur de leur modèle. La conception même de la science et le statut du scientifique amènent à un véritable questionnement sur la compréhension mutuelle entre l'explorateur et les hommes de son temps, à Paris comme dans une brousse lointaine. L'exemple d'Alfred Grandidier est particulièrement pertinent pour révéler les différentes images véhiculées sur le voyageur scientifique au XIX ${ }^{\mathrm{e}}$ siècle, et pour mettre en lumière les nombreux enjeux soulevés par la perception variée de ses travaux.

\section{Introduction}

La riche problématique de l'image de la science, du scientifique et de ses travaux nous conduit à nous interroger sur les écarts importants de perception qui peuvent exister à propos d'un même objet. C'est donc à travers l'exemple précis des voyages scientifiques d'Alfred Grandidier entre 1857 et 1870 que nous avons choisi de développer cette réflexion, pour en saisir les facettes d'une richesse mais aussi d'une complexité qui n'émergent réellement qu'à travers le croisement des points de vue. Il s'agit donc ici d'envisager la réception des recherches de terrain d'Alfred Grandidier en étudiant quatre points de vue d'époque, variés et complémentaires. On évoquera donc successivement la perception que possède le savant de ses propres recherches, le regard des autorités scientifiques et politiques sur ses résultats scientifiques, le point de vue des populations locales et enfin l'image du savant qui s'est forgée chez les successeurs d'Alfred Grandidier.

Avant d'entrer véritablement dans le vif du sujet, une rapide présentation du personnage et de sa carrière s'impose puisque sa notoriété a beaucoup décliné depuis un siècle. Alfred Grandidier (1836-1921) est un explorateur et savant français au savoir encyclopédique. Géologue, physicien et mathématicien de formation, il se passionne et s'illustre surtout en zoologie, géographie, ethnographie, mais touche aussi à l'astronomie, la botanique, la physique du globe... Favorisée par des moyens financiers très importants, cette curiosité naturelle le conduit logiquement à voyager. En effet, de 1857 à 1870, le jeune scientifique parcourt le monde:Amérique du Sud avec son frère,puis Inde du Sud, Ceylan, Zanzibar, La Réunion et surtout Madagascar, dont il devient le spécialiste incontesté pour plusieurs décennies. Il rapporte de ses périples de très nombreux spécimens d'histoire naturelle qui font sa renommée chez ses collègues naturalistes, mais également des objets ethnogra- 
phiques, des clichés et des centaines de pages de notes. De 1870 à sa mort, au contraire, Alfred Grandidier ne quitte plus les bibliothèques et laboratoires des grandes institutions scientifiques parisiennes (Muséum National d'Histoire Naturelle, Académie des Sciences, Société de Géographie...). La carrière scientifique d'Alfred Grandidier se révèle donc extrêmement longue même si actuellement l'on retient surtout la seconde partie, celle de la rédaction à Paris de dizaines d'ouvrages, dont certains titres restent célèbres chez les spécialistes de Madagascar, comme l'Histoire physique, naturelle et politique de Madagascar en quarante volumes ou encore la Collection des ouvrages anciens concernant Madagascar. La mémoire collective demeure sans doute fascinée par son impressionnante bibliographie. Il nous semble d'autant plus pertinent de nous focaliser ici sur le début de sa carrière scientifique, celle des explorations et des recherches de terrain.

\section{La vision réflexive du travail scientifique par Alfred Grandidier et la relation qu'il en fait pour autrui}

Alfred Grandidier arbore une haute opinion de lui-même, de ses travaux et de la science en général. Sa conception se rapproche souvent de la philosophie scientiste, ${ }^{1}$ bien qu'il demeure par ailleurs catholique. Il évolue dans une société fortement imprégnée par le positivisme, phénomène qui explique en partie sa passion pour la science.

A l'étude de sa vie et de son œuvre, nous percevons une franche dichotomie entre travail scientifique en amont et présentation du fruit de cette recherche en aval. Sa conception du labeur scientifique implique l'homme dans sa globalité: son intelligence, son corps et son affect. Nous entendons ce dernier terme sur le plan intime du plaisir aussi bien que sur le plan social de l'ambition. La divulgation des résultats de ce travail ressortit quant à elle à la notion de vérité. Le caractère incontestable attribué à la parole scientifique impose une désincarnation du scientifique qui doit disparaître en tant qu'être humain pour que seule émerge la connaissance.

Il est âgé de 25 ans seulement lorsqu'après deux ans de voyage en Amérique, il décide d'explorer le Tibet et de s'intéresser notamment aux traditions religieuses bouddhiques. La raison de son choix: la stimulation physique et intellectuelle. Lhassa et ses environs constituent l'une des régions les plus difficiles d'accès, encore presque inconnue à l'époque. «Le Tibet était alors inabordable [...] C'était mon affaire», ${ }^{2}$ écrit-il dans ses Souvenirs de voyages.

1 Kent 1969.

2 Grandidier, A., 1971, 5. 
Alfred Grandidier aime allier le défi physique et mental de l'exploration à la découverte scientifique. Il s'oppose en cela à la conception de l'époque selon laquelle l'aventure ne peut qu'être subie et non recherchée. ${ }^{3}$ Pour Alfred Grandidier, il n'est pas antinomique de prétendre au statut de scientifique sérieux et respecté tout en recherchant systématiquement l'expérience personnelle que représente l'aventure sur le terrain et l'inconnu. Il réussit la synthèse, très rare à l'époque de sa jeunesse, entre le parfait collecteur de terrain et l'érudit des bibliothèques. En somme, Alfred Grandidier conçoit la science comme une sorte d' «art total», une discipline de l'être dans son intégralité, physique, intellectuelle, émotionnelle, sociale.

Car Alfred Grandidier souhaite se consacrer à l'étude de régions inexplorées, dans le but précis de se forger une notoriété en s'assurant un grand nombre de découvertes tant zoologiques que botaniques ou topographiques. Les régions qui ne sont plus «vierges» géographiquement et scientifiquement ne l'intéressent pas. ${ }^{4}$ C'est tellement vrai qu'il n'envisage en 1865 qu'un simple voyage, presque touristique, à Madagascar, une île qu'il croit extrêmement bien connue des Européens. Le livre de Leguevel de Lacombe, Voyage à Madagascar et aux îles Comores, dans lequel l'auteur prétend avoir sillonné la Grande-Ile de 1823 à 1830, l'a notamment induit en erreur. La prise de conscience que la majeure partie de l'île demeure absolument méconnue et que les pseudoconnaissances à son sujet relèvent de la rumeur et des légendes, décide Alfred Grandidier à se détourner du Tibet pour se consacrer à l'étude de Madagascar.

Sa conception exhaustive, rigoureuse et méthodique de la science lui impose l'ambition d'une analyse totale de l'île. Topographie, géologie, climatologie, zoologie et paléontologie, botanique et anthropologie, aucune discipline n'échappera à son investigation. Cela se traduit par la constitution de collections complètes de spécimens d'histoire naturelle, l'enregistrement de données en séries les plus longues et les plus documentées possible.

Pour mener à bien cette mission, il retourne en France en 1867 afin de se procurer l'ensemble des instruments scientifiques indispensables à l'obtention de résultats fiables et incontestables, issus des plus récentes avancées technologiques, parmi lesquelles la photographie. Sur le terrain même, faisant fi de la fatigue ou de la maladie, il s'astreint à un travail quotidien de collecte, mesure, observation, consignant la totalité de ses notes dans de petits carnets numérotés. ${ }^{5}$

3 Venayre 2004, 63.

4 Notamment sur l'Inde britannique déjà bien connue, voir sa déception dans Grandidier, A., 1884,4 .

5 MNHN Ms 3259 à 3261, carnets de notes et d'itinéraires. 
L'image intériorisée par Alfred Grandidier concernant la présentation du travail scientifique, ennemie de la moindre fantaisie, lui vient de son père ${ }^{6}$ mais également de son mentor, l'astronome Jules Janssen. Comme ce dernier, il refuse catégoriquement de céder à l'usage de préambules narratifs aux publications de ses résultats scientifiques. D'autres explorateurs des plus sérieux comme Alexander von Humboldt ou Alcide d'Orbigny, qu'Alfred Grandidier prend par ailleurs en modèle, ont sacrifié à la tradition de la courte introduction pittoresque dans la veine du récit de voyages et d'aventures. Selon Sylvain Venayre, cette habitude remonterait chez les scientifiques et les hommes de lettres à l'Itinéraire de Paris à Jérusalem de Chateaubriand. ${ }^{7} \mathrm{~L}$ 'on sait qu'au $\mathrm{XX}^{\mathrm{e}}$ siècle, dans sa célèbre introduction de Tristes Tropiques, Claude Lévi-Strauss s'emporte à son tour contre cette pratique de se mettre en scène tel un héros d'aventures, qu'il juge nuisible à la démarche scientifique. Alfred Grandidier se montre quant à lui si extrémiste qu'il préfère ne rien écrire, comme pour son voyage en Amérique, ${ }^{8}$ que de consentir à une relation de ses aventures, surtout s'il juge ses résultats scientifiques trop maigres.

Il nous paraît que le soin extrême porté à la construction de son image préside à ce souci de ne rien laisser transpirer de sa vie quotidienne. Alfred Grandidier, de retour à Paris, cultive ce côté austère et strict, presque dégagé des contingences du monde, indispensable dans son esprit pour se garantir un statut de scientifique incontesté. Sa crédibilité scientifique et sa respectabilité bourgeoise, deux facettes de lui-même qu'il ne peut dissocier, sont également préservées par le voile pudique qu'il jette volontairement sur ses amitiés interlopes et ses amours illégitimes du temps de ses voyages. ${ }^{9}$ C'est pour cela qu'il évite les célèbres soirées d'explorateurs de la crèmerie La Petite Vache, où l'atmosphère populaire et décontractée est propice aux épanchements que le peintre Van Muyden décrit ainsi: «La Petite Vache,c'est la table d'hôtes ou plutôt la table de famille modeste d'aspect [où les explorateurs] disent les aventures intimes qu'on ne peut révéler au public de la Sorbonne.» ${ }^{10}$ Le mystère entretenu par Alfred Grandidier doit renforcer son aura scientifique.

Il n'existe que deux exceptions à cette attitude intransigeante vis-à-vis des anecdotes de voyage. La première est une erreur de jugement, dont il

6 MNHN Ms 2807, Grandidier, A., 1917. Il raconte notamment les dures heures d'étude dès son plus jeune âge et l'éducation d'inspiration janséniste qu'il a reçue.

7 Venayre 2004, 62.

8 Il laisse ce soin à son frère. Grandidier, E., 1861.

9 Ces secrets sont bien gardés tout au long de sa vie et n'apparaissent qu'à la faveur de courriers personnels. Voir archives MNHN, notamment Ms 2811 à 2823.

10 www.brazza.culture.fr 
gardera toute sa vie un goût amer. Il consent à publier une relation de son séjour en Inde dans la célèbre et populaire revue française du Tour du Monde. A notre connaissance, c'est l'unique circonstance où il ne publie pas dans une revue scientifique. Afin de rendre plus attrayante une étude sèche et érudite, enrichie de multiples références sur le bouddhisme et ses origines, il accepte de raconter les détails de son périple à travers le sous-continent. Il ne tarde pas à le regretter lorsqu'il constate qu'il s'est trompé de public et que sa démarche ne peut être comprise, ni par la rédaction, ni par le lectorat. En témoigne cette réflexion du scientifique:

M. Lannoy, qui dirigeait cette publication, a trouvé bon de supprimer la plus grande partie de mon récit relatif à la religion bouddhique qu'il a trouvée trop scientifique et trop sévère pour ses lecteurs. Et moi qui n'avais écrit cette relation de mon voyage dans l'Inde que pour faire passer cette étude sur le Bouddhisme!11

Une telle méprise, dont on peut s'étonner de sa part, suscite une déconvenue si profonde que les innombrables publications qui suivent restent exclusivement scientifiques.

Nous pouvons cependant déceler une deuxième exception dans le volume de sa monumentale Histoire Physique, Naturelle et Politique de Madagascar, ${ }^{12}$ consacré aux mammifères. Au milieu de pages exclusivement dévolues à la description des espèces, Alfred Grandidier raconte en détail sa mésaventure lorsqu'il tue son premier lémurien, reprenant un texte déjà paru à La Réunion en 1867. ${ }^{13}$ Nous émettons l'hypothèse de la relation de cette mésaventure dans un souci de contextualisation de la chasse, bien que le texte insiste davantage sur les difficultés du chasseur plutôt que sur le lémurien! Il s'agit sans doute d'un exemple des nombreux paradoxes du scientifique.

Nous ne classons pas dans la même catégorie la petite autobiographie qu'il rédigea en $1917,{ }^{14}$ déjà très âgé et quasiment retiré des affaires scientifiques, à la demande de ses enfants et petits-enfants, comme il le précise en introduction. Ce texte, à usage familial et non scientifique, est d'ailleurs resté inédit, sauf la partie concernant Madagascar publiée en $19711^{15}$ puis un extrait concernant les Amériques cité en 2005. ${ }^{16}$

En dernier lieu, nous évoquerons l'absence de réflexion épistémologique, dans son acception de la tradition philosophique francophone, de la part d'Alfred Grandidier. C'est une attitude qui ne tranche pas avec le comportement de la plupart des scientifiques de son temps. A aucun moment, Alfred

11 Grandidier, A., 1971, 5.

12 Grandidier, A., 1875, 294.

13 Grandidier, A., 1866-1867.

14 MNHN, Ms 2807.

15 Grandidier, A., 1971.

16 Verin 2005. 
Grandidier ne se questionne sur la validité ou le bien-fondé de son entreprise. Jamais il ne se départit de son attitude surplombante d'Européen civilisateur et ne cesse de porter des jugements moraux sur les peuples qu'il étudie. ${ }^{17}$

\section{L'appréciation des autorités scientifiques et politiques sur les résultats scientifiques d'Alfred Grandidier}

S'il ne publie quasiment jamais le récit de son voyage lors de son retour d'exploration, Alfred Grandidier s'empresse en revanche de faire bénéficier les autorités scientifiques, voire politiques, de la primeur de ses travaux par de nombreuses communications orales et publications scientifiques. Il s'agit pour lui de gagner l'estime et la reconnaissance du monde scientifique et politique et non d'obtenir, comme tant d'autres, des subventions convoitées puisque l'explorateur s'autofinance entièrement. Cette particularité représente un atout inestimable dans sa carrière. S'il avait dû compter sur l'appréciation immédiate de ses découvertes scientifiques pour réunir les fonds nécessaires à de nouvelles expéditions, il n'aurait peut-être pas poursuivi les recherches qui l'ont finalement rendu célèbre.

Les honneurs et les récompenses dont il fut gratifié lors de ses retours de voyages masquent une méconnaissance de la qualité véritable ainsi que du caractère novateur de ses travaux. Ses contemporains tendent à valoriser les aspects directement utiles de ses recherches, ignorant le caractère annexe aux objectifs primordiaux d'Alfred Grandidier des travaux et collectes qu'ils privilégient.

Pour illustrer le pragmatisme dont fait preuve la société nous citerons l'exemple de l'acclimatation d'animaux ou de végétaux. Dès son retour en France en 1867, après deux expéditions dans l'est et le sud de Madagascar, il se trouve en phase avec les préoccupations coloniales françaises en favorisant l'acclimatation en Algérie des martins tristes de La Réunion, ${ }^{18}$ notoires chasseurs de criquets et autres sauterelles. Pour cette introduction, Alfred Grandidier est chaudement félicité et entretient une correspondance avec l'empereur Napoléon III, très intéressé par les détails de cette naturalisation

17 Bien qu'il se présente toujours comme neutre et extérieur car homme de science, Alfred Grandidier ne peut par exemple s'empêcher d'intervenir pour sauver un nourrisson malgache de la mort, condamné par la tradition villageoise parce qu'il est né un jour néfaste. Non seulement il juge la culture qu'il étudie, mais il intervient et prend prétexte de cet exemple pour en tirer une leçon de morale. Grandidier, A., 1971,11.

18 Ce n'est même pas une espèce endémique ou méconnue. Les acridotheres tristis avaient été introduits à La Réunion par Pierre Poivre à la fin du XVIII ${ }^{\mathrm{e}}$ siècle pour chasser les sauterelles. N'importe qui aurait donc pu avoir l'idée de les acclimater en Algérie. 
qui doit aider au développement agricole de la colonie française. Alfred Grandidier est ensuite chargé officiellement par Napoléon III et le Gouverneur d'Algérie de procéder à l'introduction d'autres oiseaux réunionnais en Algérie, très importante terre d'acclimatation au XIX ${ }^{\mathrm{e}}$ siècle. ${ }^{19}$

Déjà lors du voyage en Amérique du Sud, Alfred Grandidier était salué pour son acclimatation du chenopodium quinoa et de vingt nouvelles espèces de maiis andins par la Société d'Acclimatation qui lui décerna la Grande Médaille d'Or. S'il était remercié par le Ministre de l'Instruction Publique, c'était pour avoir contribué à valoriser l'image de la France à l'étranger ${ }^{20}$ Les journaux diffusèrent à peu près le même discours, très vague: «Messieurs Ernest et Alfred Grandidier ne pouvaient faire plus noble emploi de leur fortune que de la consacrer à des travaux qui sont, en résumé, un bénéfice pour la science et un honneur pour leur pays.» ${ }^{21}$

Peu de publicité est donnée sur le moment à ses travaux astronomiques ou à ses découvertes en Histoire Naturelle. Qui reconnaît, hors de la Sorbonne, la valeur des nouvelles collections de plantes, reptiles et minéraux qui font sa fierté ${ }^{22}$ De même, les contributions majeures du jeune scientifique à Madagascar concernant les corrections cartographiques, la différenciation entre les singes et les lémuriens, l'étude de fossiles d'animaux encore mystérieux, la genèse de la formation géologique de l'océan Indien, ou l'estimation de la richesse et du taux d'endémicité exceptionnel de la flore malgache, passent presque inaperçues hors des milieux les plus savants comme l'Académie des Sciences ${ }^{23}$, alors qu'elles constituent l'essence de son objectif et lui ont demandé de longues années de réflexion et de développement. De même, les autorités militaires n'utilisent les données cartographiques de l'explorateur que plus de vingt ans après le début de leur publication. ${ }^{24}$

\section{La perception des recherches scientifiques d'Alfred Grandidier par les populations autochtones}

Les autorités françaises ont sans doute manqué de clairvoyance immédiate quant à la portée des travaux scientifiques d'Alfred Grandidier. En revanche,

19 Brockway 1979, 58.

20 Decary 1936.

21 MNHN, article paru dans Le Moniteur le 3 août 1860 et dans Le Journal Général de l'Instruction Publique le 4 août 1860.

22 Grandidier, A., 1884, 45.

23 Grandidier, A., 1884.

24 La première carte de l'Imerina est parue en 1871, la conquête militaire n'a été lancée qu'à la fin de l'année 1894. 
sur le terrain, les populations locales, notamment les Malgaches, semblent avoir perçu le considérable impact sur leur existence ainsi que les menaces potentielles induits par ses recherches et observations. S'il n'est pas aisé de connaître la pensée des autochtones, dont la voix n'est quasiment relayée que par le truchement des notes d'Alfred Grandidier, il est avéré que l'explorateur a été plus d'une fois considéré avec méfiance, voire hostilité, d'autant plus qu'il lui arrivait d'enfreindre les croyances et coutumes locales. Un certain nombre de sources écrites émanant de l'Etat merina témoignent de relations tendues et d'une suspicion mutuelle. ${ }^{25}$ Cependant, nous n'ignorons pas que Marshall Sahlins a brillamment démontré dans La découverte du vrai Sauvage que l'hétéroglossie peut conduire à des analyses totalement contradictoires d'une même situation historique. ${ }^{26}$ Cela nous invite à la prudence.

D'une manière générale, Alfred Grandidier est plutôt mal reçu à Madagascar, aussi bien lors de ses activités d'investigation que pour l'usage d'instruments sophistiqués. Il est très rapidement considéré comme un sorcier dans le sud et l'ouest de l'île, une accusation grave qui peut lui valoir la mort. Nous signalons toutefois que cette situation, loin d'être spécifique à Madagascar, menace tous les scientifiques-voyageurs, quelle que soient leur destination et leur époque.

Concernant le premier grief sur les collectes d'objets d'Histoire Naturelle, le scientifique commet un grave impair dès le début de son second voyage. Il tue un lémurien $f a d y$, c'est-à-dire tabou, sacré, provoquant la colère de villageois des environs. Afin de réparer l'offense, le chef local fait procéder à l'enterrement de l'animal, autorisant tout de même Alfred Grandidier à conserver la peau en vue de la naturalisation du spécimen. Cependant, le voyageur doit quitter les lieux précipitamment.

Le deuxième motif impliquant l'usage des instruments de l'explorateur suscite davantage encore la méfiance, pour deux principales raisons. L'une est d'ordre magique. Les Malgaches prêtent des pouvoirs néfastes à ce matériel, par exemple à l'appareil photographique, ainsi que le raconte, quelque peu dépité, Alfred Grandidier: «Quand je faisais la photo de l'un d'eux, je lui 〈volais son âme〉 et dans l'Ouest beaucoup s'enfuyaient quand ils me voyaient mettre la chambre noire sur mon trépied. $»^{27}$ Notons encore une fois que la photographie, tout particulièrement, prête souvent à malentendu entre voyageurs et populations locales du monde entier. ${ }^{28}$

25 Verin 1971.

26 Sahlins 2007, 141.

27 Grandidier, A. 1971, 21.

28 Fournié 2003, 29. 
La seconde raison relève de la stratégie politique. Les Malgaches du Sud, observant le théodolite, la lunette astronomique ou encore la boussole, instruments qu'ils ne connaissent pas, saisissent aussitôt que cela sert, selon leur expression, à «mettre le pays sur papier» ${ }^{29}$. D'après l'explorateur, les Malgaches associent ses activités scientifiques avec un potentiel risque de conquête militaire. «Est-ce que ce Blanc n'inspecte pas notre pays pour nous faire la guerre? $\gg^{30}$, s'interroge-t-on sur son passage. En la matière, les Malgaches font preuve d'une clairvoyance sur les implications des recherches d'Alfred Grandidier qu'il ne possède pas lui-même à cette époque. Claude Blanckaert rappelle que les géographes du $\mathrm{XIX}^{\mathrm{e}}$ siècle se sont souvent impliqués dans le renseignement, voire la diplomatie. ${ }^{31}$ Les Malgaches ne s'y trompent pas, même si l'explorateur ne travaille pas, à cette date, pour l'armée française. A la fin de sa vie, alors que Madagascar appartient à l'empire colonial français depuis 20 ans, le scientifique reconnaît la grande perspicacité des Malgaches:

Un grand nombre d'indigènes ont attribué notre conquête aux instruments mystérieux et cabalistiques pour eux de topographie et de météorologie qu'ils me voyaient consulter [...] chaque jour, à toute heure, et leurs craintes n'étaient pas si folles puisque les cartes que j'ai levées à l'aide de ces instruments n'ont pas été sans avoir eu leur utilité pour la conquête de leur pays. ${ }^{32}$

Il corrobore ainsi l'avis d'un historiographe de la Grande Ile, le Capitaine De Vilars, qui écrivait en 1912:

Il convient de citer les magnifiques explorations de M. Alfred Grandidier à Madagascar de 1865 à 1870. Bien qu'elles n'aient eu aucun caractère politique, elles ont apporté aux Français tant de connaissances sur leur future colonie qu'elles les ont incontestablement aidés à s'y établir. ${ }^{33}$

Dans l'ouest de Madagascar, haut-lieu de traite négrière, l'explorateur est assimilé à un espion à la solde des abolitionnistes britanniques et dont les activités scientifiques ne seraient qu'un prétexte. Sa vie est alors menacée par les négriers malgaches et musulmans qui voient leurs traditions et leurs intérêts commerciaux contrecarrés. Sur le moment, Alfred Grandidier se défend de l'accusation d'espionnage et réaffirme le caractère purement scientifique et indépendant de sa situation. Quelques années plus tard, il avoue avoir fourni des renseignements aux autorités britanniques, leur permettant de faire reculer significativement la traite dans cette zone au cours des mois suivants. ${ }^{34}$ En fait, l'on peut remarquer la perpétuelle ambi-

29 Grandidier, A., 1971, 21.

30 Grandidier, A., 1971, 26.

31 Blanckaert 2008, 28.

32 Grandidier, A., 1971, 21.

33 De Vilars 1912, 234.

34 Grandidier, A., 1971, 27.

Gesnerus 70 (2013) 
guïté avec laquelle Alfred Grandidier joue avec son statut de scientifique. Selon les circonstances, il se présente comme indépendant de toute autorité politique, mais investi par la hauteur du but poursuivi d'une aura presque sacrée, ou inversement comme un envoyé de l'empereur et digne du respect réservé aux ambassadeurs. ${ }^{35}$

L'ambivalence de son attitude contribue donc à brouiller la perception qu'ont de lui les populations locales. Il ne semble pas adéquat de déduire l'opposition quasi systématique des Malgaches aux activités scientifiques d'Alfred Grandidier d'un obscurantisme à outrance, comme l'explorateur ne cesse de le dénoncer dans ses notes, ${ }^{36}$ mais bien d'une crainte fondée au regard des divers impacts de son travail.

\section{L'image du scientifique de terrain chez les continuateurs de l'œuvre d'Alfred Grandidier}

Contrairement aux peuples autochtones, au premier rang desquels les Malgaches, les scientifiques ont quasiment tous encensé l'œuvre d'Alfred Grandidier. Chez ses héritiers directs, l'on peut même parfois parler de véritable hagiographie. Unanimement vanté pour la qualité comme pour la quantité de ses travaux, il fait à peu près l'objet d'une «héroïsation» du scientifique de terrain. Jusqu'à nos jours, Alfred Grandidier reste l'archétype du scientifique explorateur, ainsi qu'on peut le lire sous la plume d'Yvan Paillard, en 1992: «Aucun [explorateur de Madagascar] n'égale en mérite, en audace tranquille et en qualité scientifique Alfred Grandidier.» ${ }^{37}$

$\mathrm{Au}$ fil du temps se construit une véritable légende autour de la figure d'Alfred Grandidier en brousse. Raymond Decary, bien qu'il trace également des portraits plus nuancés, apparaît comme l'un des principaux contributeurs à une vision tout d'abord romantique du scientifique. Alimentée par le propre fils d'Alfred Grandidier, ${ }^{38}$ reprise et colportée ensuite par d'autres, ${ }^{39}$ elle entretient l'image d'un explorateur aux pieds nus, qui arpente les sentiers et fait corps avec la terre qu'il étudie, à l'instar des Malgaches eux-mêmes. ${ }^{40}$

La dimension du personnage devient plus mystique lorsque à cette humilité supposée se trouvent associés des détails qu'Alfred Grandidier distille

35 Grandidier, A., 1971, 13, 18, 24, 27, 37, 46.

36 MNHN Ms 3259, notamment carnet $\mathrm{n}^{\circ} 2$.

37 Paillard, 1994, 290.

38 Grandidier, G., 1946.

39 Poisson 1938, 50.

40 Decary 1936. En poursuivant la métaphore religieuse, on pourrait parler ici d'image christique appliquée au savant voyageur. 
lui-même à la fin de sa vie auprès de ses collaborateurs. Ces informations portent le plus souvent sur les difficultés et les contraintes liées à ses recherches de terrain: privations, souffrances, précarité des conditions matérielles. Inconsciente ou non, cette attitude favorise l'émergence d'une figure doloriste quasi religieuse. L'explorateur est assimilé à un missionnaire de la science et du progrès. Ce terme contient les connotations de la mortification, voire du martyre. ${ }^{41}$ Il peut être associé au stéréotype du «héros de la géographie» en contexte colonial, tel que défini par Morag Bell, Robin Butlin et Michael Heffernan: un homme en butte à l'hostilité des indigènes et des éléments naturels. ${ }^{42}$

La dernière étape établit une mythologie complète autour d'Alfred Grandidier. Du voyageur passionné en passant par l'homme qui s'est sacrifié, nous en arrivons au héros qui, dans la plus grande solitude, aura su braver toutes les adversités à la recherche de la vérité. Il paraît donc utile d'en revenir aux faits. Nous avons souligné l'originalité d'Alfred Grandidier: l'association d'une parfaite maîtrise de terrain à de multiples connaissances scientifiques qui lui permet un travail analytique des collectes doublé d'une vaste entreprise de publication. Jusqu'aux dernières décennies du XIX ${ }^{\mathrm{e}}$ siècle, la dichotomie était restée de règle entre le collecteur de terrain et le scientifique de cabinet.

Cependant, le désir de rester fidèle autant que possible à la réalité, sans pour autant amoindrir les performances vraiment remarquables d'Alfred Grandidier, nous conduit à préciser quelques points. S'il est vrai qu'il parcourt une grande partie du sud-ouest malgache pieds nus - se plaignant d'ailleurs des nombreuses épines et du sable brûlant ${ }^{43}-$, il est exagéré de prétendre, comme le fait son fils ${ }^{44}$ que c'est là son mode de déplacement par excellence. Il utilise la chaise à porteurs, le filanjana, à diverses reprises. ${ }^{45}$

41 Malgré tout le bonheur qu'il tire de ses voyages et de ses recherches, l'association fréquente chez Alfred Grandidier entre souffrances physiques et pratique de la science sur le terrain est peut-être à rapprocher de son enfance très marquée par le jansénisme. Par ailleurs, Alfred Grandidier se considère comme une sorte de missionnaire qui, non content d'étudier les régions reculées, doit leur faire partager sa foi en la science comme source de progrès pour l'Humanité et pour leurs peuples en particulier. Il se plaint très souvent d'être incompris sur ce point des populations locales. Decary 1936,12. Il n'est guère exagéré de parler de martyr non plus quand on sait à quel point Alfred Grandidier a toujours veillé à promouvoir le souvenir de ses successeurs morts en mission, principalement dans les années 1890, qu'ils aient trépassé suite à la maladie comme Douliot ou à des attaques comme Müller. Grandidier, A. 1893. A l'aune des difficultés rencontrées sur le terrain par les jeunes scientifiques au tournant du XX $\mathrm{X}^{\mathrm{e}}$ siècle, ses contemporains réévaluent les épreuves qu'a dû traverser Alfred Grandidier lui-même, ses travaux de terrain n'en sont que davantage admirés et glorifiés.

42 Bell 1995, 4.

43 Grandidier, A., 1971, 15, 29.

44 Grandidier, G., 1946.

45 Grandidier, A., 1971, 44, et MNHN Ms 3259, carnet nº 8. 
Lors de ses voyages aux Amériques et en Inde, il liste une imposante quantité de modes de transports et ne dédaigne pas les moyens les plus modernes et les plus confortables, tels le chemin de fer.

Notre restriction concerne également le caractère solitaire de ses explorations. Il se fait accompagner en permanence d'au moins 3 domestiques à Madagascar. Outre les Malgaches, des traitants, tels Edmond Samat dans l'Ouest, ou des missionnaires comme le Père Roblet dans le Centre, partagent ses déplacements. En Amérique de même, il est accompagné de son frère et d'au moins un domestique. En Inde, il ne se déplace jamais sans son boy, et rencontre fréquemment des Européens lors de ses étapes. ${ }^{46}$

La légende d'Alfred Grandidier se nourrit également de la confusion, entretenue par lui-même puis par ses successeurs, au sujet de l'étendue de ses relevés et collectes sur le terrain. On lui a volontiers tout attribué, notamment les auteurs d'articles courts ou non spécialisés. Or, il a reconnu publiquement, quoique assez tard, que les premières cartes qu'il avait fait publier en 1871-1872, par exemple, devaient bien davantage aux relevés et calculs du Père Roblet qu'aux siens. C'est donc seulement dans les années 1890 que le jésuite connaît une gloire aussi légitime que relative. ${ }^{47}$ De même, nombre de collections d'Histoire Naturelle n'ont pas été constituées personnellement par Alfred Grandidier lors de ses voyages mais par des relais sur place alors qu'il avait définitivement regagné Paris. Ces contributeurs, des colons et souvent des religieux, s'ils bénéficient de toute l'estime d'Alfred Grandidier - nous le constatons dans les courriers ou dans les dénominations d'espèces nouvelles - sont restés absolument anonymes pour la plupart d'entre eux. ${ }^{48} \mathrm{De}$ ce fait, au décès de l'explorateur, l'ensemble des observations, relevés et collections est porté à son crédit et souvent rendu public sous son nom. Il était entre-temps devenu une sorte de label, «l'Homme de Madagascar» selon l'expression consacrée. ${ }^{49}$

Nous terminerons en évoquant l'attitude de ses successeurs, au premier rang desquels son fils Guillaume, et l'ami de celui-ci Raymond Decary, qui ont présenté le savant en chantre de la colonisation de Madagascar. Decary assure qu'Alfred Grandidier connut la satisfaction de voir la Grande Ile colonisée et qu'il fut «un des meilleurs ouvriers de la cause coloniale». ${ }^{50}$ A l'apogée de la période coloniale, l'on concevra qu'il est tentant pour ses

46 Grandidier, E., 1861, et Grandidier, A., 1868.

47 Plusieurs allocutions en ce sens à l'Académie des Sciences, notamment les rapports de remises de prix.

48 Dans l'Histoire naturelle des plantes de Madagascar, le nom des collecteurs est systématiquement mentionné cependant. Mais Alfred Grandidier n'en est pas directement l'auteur.

49 Lacroix 1922, 37.

50 Decary 1938, 12. 
héritiers de récupérer cette figure emblématique. Un jugement plus mesuré semble de mise en l'occurrence. Les sentiments d'Alfred Grandidier à l'égard de la colonisation de Madagascar ont eu le temps d'évoluer au fil des décennies. Il est certain, par exemple, qu'il n'a pas voyagé en vue de favoriser une future colonisation de Madagascar. A l'inverse, après 1960 et l'indépendance malgache, occulter le rôle direct du savant au moment de la conquête de l'île, c'est pécher par omission. ${ }^{51} \mathrm{Il}$ est incontestable que le scientifique a mis à disposition de l'armée et du gouvernement toutes les informations qu'il avait pu recueillir sur le terrain, ainsi que ses conseils. ${ }^{52}$

\section{Conclusion}

Au terme de cette étude de cas sur le sujet central de l'image du scientifique, nous préciserons que la légende créée autour des explorations d'Alfred Grandidier s'est trouvée si distordue que ses véritables recherches en ont souffert. En effet, si le renom d'Alfred Grandidier peut à juste titre être lié à Madagascar, cette célébrité réductrice passe sous silence ses découvertes en Amérique du Sud aussi bien que ses recherches en Asie et à Zanzibar. Cumulées, les durées de ses autres voyages sont sensiblement équivalentes à celle de ses explorations malgaches. ${ }^{53}$ Bien que concentrant son énergie sur la rédaction de ses ouvrages sur Madagascar, Alfred Grandidier n'a jamais cessé de porter un intérêt particulier aux travaux réalisés dans d'autres contrées, de la Russie à l'Afrique centrale.$^{54}$ La carrière scientifique d'Alfred Grandidier recèle bien davantage de richesse et de variété qu'on ne le pense communément. Des pans entiers sont aujourd'hui tombés dans l'oubli: sa collection d'objets ethnographiques amérindiens, ses études architecturales de temples en Inde et à Ceylan, sa curiosité pour les nouveautés technologiques au service de l'exploration ou encore son appui aux missionnaires et naturalistes en Afrique centrale.

Cette étude doit donc contribuer à dévoiler succinctement aussi bien la richesse que la complexité ou l'ambiguïté, qui augmentent en proportion du croisement des points de vue portés sur cette carrière scientifique intéressante parce qu'éclectique. C'est en présentant une telle mosaïque des regards

51 Exposition commémorant le centenaire des voyages à Madagascar d'Alfred Grandidier, Académie Malgache, Tananarive, 1970-1971.

52 Julien 1922.

53 Environ trois ans passés à Madagascar entre 1865 et 1870 et trois ans et demi passés aux Amériques, en Inde et à Zanzibar.

54 Voir le soutien apporté à la mission française en Afrique centrale et orientale et au général Tilho pour la carte de la Russie centrale et orientale, Bulletin de l'Académie des Sciences. 
que nous participons d'un renouvellement et d'un élargissement de l'éclairage focalisé sur l'image parfois figée ou stéréotypée d'un scientifique comme Alfred Grandidier. Chaque regard porte sa propre partialité et son incomplétude, y compris le sien. Seule leur confrontation nous permet d'envisager la globalité des enjeux induits par une pratique scientifique de terrain.

\section{Abréviation}

MNHN: Archives et Bibliothèque du Muséum National d'Histoire Naturelle de Paris.

\section{Bibliographie}

Bell, Morag/Butlin, Robin/Hefferman, Michael, Geography and imperialism, 1820-1940 (Manchester 1995)

Blanckaert, Claude, «Eux et nous: l'Empire des Sciences», dans L'empire des géographes: géographie, exploration et colonisation $X I X^{e}-X X^{e}$ siècles (Paris 2008) 27-44

Bourguet, Marie-Noëlle, Instruments, Travel and Science. Itineraries of precision from the seventeenth to the twentieth century (Londres 2002)

Broc, Numa, «Les explorateurs français du XIX ${ }^{\mathrm{e}}$ siècle reconsidérés», Revue française d'Histoire d'Outre-Mer 256 (1982) 236-272

Brockway, Lucile, Science and colonial expansion. The role of the British Royal Botanic Gardens (New York 1979)

Collectif, L'empire des géographes: géographie, exploration et colonisation XIX ${ }^{e}$ $X X^{e}$ siècles (Paris 2008)

Decary, Raymond, «Alfred Grandidier, explorateur de Madagascar», Revue de Madagascar 16 (1936) 1-35

Decary, Raymond, «A la mémoire de l'explorateur Alfred Grandidier, lettre ouverte au Président du Comité de Madagascar», Revue de Madagascar 22 (1938) 9-16

De Vilars, Capitaine, Madagascar 1638-1894, Etablissement des Français dans l'île (1912)

Fournié, Pierre/de Sivry, Sophie, Aventuriers du monde, les grands explorateurs français au temps des premiers photographes (1866-1914) (Paris 2003)

Grandidier, Alfred, «Types nouveaux ou peu connus du Muséum de Saint-Denis Le propithèque de Verreaux», dans Roussin, Louis-Antoine, Album de l'Ile de La Réunion (Saint-Denis-de-La Réunion 1866-1867) 153-162

Grandidier, Alfred, «Voyage dans les provinces méridionales de l'Inde», Le Tour du Monde (1868)

Grandidier, Alfred, Notice sur les travaux scientifiques d'Alfred Grandidier (Paris 1884)

Grandidier, Alfred, Manuscrit autobiographique inédit (MNHN Ms 2807,1917)

Grandidier, Alfred et alii, Histoire Naturelle des Mammifères, Histoire Physique, Naturelle et Politique de Madagascar (Paris 1875) 
Grandidier, Alfred, «M. Georges Müller et sa mort à Madagascar», Comptes-rendus de séances de la Société de Géographie (Paris 1893) 388-393

Grandidier, Alfred/Association d'Archéologie, Souvenirs de voyages, manuscrit de 1916 (Antananarivo 1971)

Grandidier, Ernest, Voyage dans l'Amérique du Sud, Pérou et Bolivie (Paris 1861)

Grandidier, Guillaume, «Alfred Grandidier vu par son fils», Journal des voyages (1946)

Julien, Gustave, «Alfred Grandidier 1836-1921», Revue Scientifique, 7 (1922)

Kent, Raymond, «Alfred Grandidier et le mythe des fondateurs d'états malgaches d'origine asiatique», Bulletin de Madagascar, 277-278 (1969) 603-615

Lacroix, Alfred, «Notice historique sur Alfred Grandidier lue en séance publique annuelle le 18 décembre 1921», Bulletin de l'Académie des Sciences (Paris 1922)

Lejeune, Dominique, Les sociétés de géographie en France et l'expansion coloniale au XIX $X^{e}$ siècle (Paris 1993)

Paillard, Yvan, «De l'exploration à la reconnaissance: Madagascar dans la seconde moitié du XIX ${ }^{\mathrm{e}}$ siècle», dans Découvertes et explorateurs, actes du colloque international de Bordeaux, 12-14 juin 1992 (Paris 1994) 289-300

Poisson, Charles, «Alfred Grandidier géographe», Revue de Madagascar, 22 (1938) 49-56

Raison, Jean-Pierre, «Discours scientifique et manipulation politique: les Européens face aux Merina et à l'Imerina de la fin du XVIII ${ }^{e}$ siècle aux lendemains de la conquête», dans Nordman, Daniel/Raison, Jean-Pierre, Sciences de l'homme et conquête coloniale. Constitution et usages des sciences humaines en Afrique (XIX ${ }^{e}-X X^{e}$ siècles) (Paris 1980) 23-45

Sahlins, Marshall, La découverte du vrai Sauvage et autres essais (Paris 2007)

Sibeud, Emmanuelle, Une science impériale pour l'Afrique? La construction des savoirs africanistes en France 1878-1930 (Paris 2002)

Surun, Isabelle, Géographies de l'exploration. La carte, le terrain et le texte (Afrique occidentale 1780-1880), thèse (Paris 2003)

Surun, Isabelle, «Du texte au terrain: reconstituer les pratiques des voyageurs (Afrique occidentale, 1790-1880)», Sociétés et représentations 21 (2006) 213-223

Venayre, Sylvain, «Qu'est-ce-que l'éloignement? L'Aventure, l'ethnographie et les blancs de la carte (1850-1940)», dans Isabelle Laboulais-Lesage (dir.), Combler les blancs de la carte (Strasbourg 2004) 61-87

Verin, Pierre, «Quelques lettres de l'Administration royale au sujet d'Alfred Grandidier», Bulletin de l'Académie Malgache 49/1 (1971)

Verin, Pierre, «Alfred Grandidier voyage en Amérique», dans Veccella, Robert/ Verin, Pierre, L'Amérique hispanique et le Pacifique, hommage à Hugo Neira (Paris 2005) 177-200 